\title{
Effect of reducing taxonomic resolution on ordinations to detect pollution-induced gradients in macrobenthic infaunal assemblages
}

\author{
M. A. Vanderklift*, T. J. Ward, C. A. Jacoby \\ CSIRO Division of Fisheries, PO Box 20, North Beach, Western Australia, 6020, Australia
}

\begin{abstract}
Researchers often encounter taxonomic problems when analysing data on changes in macrobenthic assemblages due to pollution. In response, some authors have suggested that data at family and even phylum level may be sufficient to detect such changes. This hypothesis is commonly tested by visual comparisons of ordination plots generated by multidimensional scaling (MDS). Our study assessed this approach using a dataset previously shown to contain patterns in species composition that were related to both habitat type and heavy metal pollution. These data allow us to assess the ability of analyses at reduced taxonomic resolutions to detect 'signals' associated with pollution gradients among the 'noise' associated with habitat gradients. Such situations will arise in most studies of the impacts of pollution on inshore macrobenthic assemblages. The patterns associated with both habitat and pollution gradients were visible in ordinations based on data pooled to higher taxonomic levels. The similarity among ordinations based on different sets of pooled data was not fully supported by more detailed analyses. Changing the number of dimensions in ordinations, the taxonomic resolution, the attribute:object ratio and the non-zeros ratio of datasets all affected the matrix of association measures and the results of ordinations and Procrustes rotations. The effects of these changes were complex. Further work is needed to determine the likely effect of using data with reduced taxonomic resolution affect to detect impacts.
\end{abstract}

KEY WORDS: Taxonomic resolution - Macrobenthos - Infauna Semi-strong hybrid multidimensional scaling · Procrustes rotation - Dataset characteristics - Effects of pollution

\section{INTRODUCTION}

Studies of anthropogenic impacts on marine environments often use benthic macrofaunal assemblages as indicators (Warwick 1993), because they can be sampled quantitatively, are small and have relatively short lifespans. Unfortunately, identifying benthic macrofauna to species is time-consuming and requires considerable expertise. As a result, rigorous studies of this type can be too expensive for widespread use.

In response, recent studies have examined ways of improving the cost-effectiveness of benthic sampling by identifying macrofauna to higher taxonomic levels than species (Ellis 1985, Warwick 1988a, b, Ferraro \& Colc 1990, Warwick et al. 1990, Agard of al. 1993. Rees 1993, Warwick \& Clarke 1993). If samples are taken

·E-mail: mat.vanderklift@per.ml.csiro.au from a single habitat, these authors suggest that analyses with less taxonomic resolution result in little loss of information about impacts and that such analyses may yield better discrimination of patterns associated with impacts. For example, natural environmental variables (e.g. water depth and sediment grain size) may affect the distribution patterns of species but not the distribution patterns of higher taxonomic groups (Warwick 1988a); therefore, the use of species-level data may introduce 'noise' into analyses designed to detect impacts. If higher taxonomic levels do respond more clearly to anthropogenic influences, it is probably better to analyse more samples at lower taxonomic resolutions than fewer samples identified to species level (Warwick 1993, Warwick \& Clarke 1993).

Before the use of lower taxonomic resolutions is widely adopted, it is important to understand the uncertainty associated with analyses at lower taxonomic 
resolutions. It is particularly important to determine the extent to which analyses at lower taxonomic resolutions recover the details contained in analyses of species-level data. This is especially true for areas that have gradients associated with factors other than pollution (e.g. habitat or depth gradients).

Macrobenthic studies commonly rely on ordinations to highlight spatial and temporal differences in assemblages, attempting to identify likely causal links by relating these differences to anthropogenic inputs. Although several recent studies have used ordination techniques such as Principal Components Analysis (e.g. Roper et al. 1989) or Principal Coordinates Analysis (e.g. Stull et al. 1986), most authors have preferred multidimensional scaling (MDS; e.g. Gray et al. 1988, Austen et al. 1989, Zenetos \& Papathanassiou 1989, Gray et al. 1990, Agard et al. 1993)

In many previous studies, authors evaluated the similarily of pditerns in ordination plots based on different levels of taxonomic resolution without the aid of statistics that directly compared the patterns (Heip et al. 1988, Warwick 1988a, b, Rees 1993, Warwick \& Clarke 1993). Where patterns appeared to change little, authors concluded that there had been little loss of information. Such subjective comparisons may suffer from observer biases including level of experience (Gray et al. 1988).

This study uses statistical methods to document changes in ordination plots that are based on macroinfaunal distributions that have been grouped at different taxonomic resolutions. Distribution patterns are expected to relate to a known gradient in metal pollution, because the samples were collected in Upper Spencer Gulf, where the sediments have high concentrations of heavy metals (Ward \& Young 1981). Previous reports have described the relationships between concentrations of metals and compositions of epifaunal and infaunal assemblages near a smelter (Ward \& Young 1982, Ward \& Hutchings 1996, this issue). In this paper, we explore whether analyses of macroinfaunal data at decreasing levels of taxonomic resolution identify patterns associated with habitat differences and pollution that are similar to those discussed by Ward \& Hutchings (1996).

We examined the changes at different taxonomic resolutions by correlating the association matrices that underlie the ordinations, by comparing stress values to determine how well ordination plots displayed a pattern, and by using Procrustes rotation to compare patterns in the ordinations. We also manipulated the data in an attempt to simulate the 2 purely numerical differences associated with data matrices at different taxonomic resolutions. In general, data matrices at higher taxonomic levels will have fewer taxa (attributes) relative to the number of samples (objects). We have termed this a change in the attribute:object ratio. In addition, most samples will contain representatives of higher taxonomic levels; therefore, the number of non-zero values per column increases as the taxonomic resolution decreases. We have termed this a change in the non-zeros ratio. Both of these changes usually accompany pooling of species-level data to higher taxa, but we attempted to examine their effects independently.

\section{METHODS}

Original dataset. The dataset consisted of macroinfaunal abundances in samples from Upper Spencer Gulf, South Australia. Ward \& Young (1981) and Ward \& Hutchings (1996) have described how these data were collected. A total of 372 species were collected in 9 replicate samples taken from each of 6 habitats along 3 transects during the austral winter of 1979 and the austral summer of 1980 . We pooled replicate samples as did Ward \& Hutchings (1996). This procedure yielded a data matrix of 36 samples ( 2 times $\times 3$ transects $\times 6$ habitats) by 372 species. As is characteristic of macroinfaunal datasets, most values in the matrix were zeros. Ward \& Hutchings (1996, this issue) reduced the dataset to 233 species by removing 'rare' species (i.e. species represented by only 1 or 2 individuals). We also removed any taxa in each dataset that were represented by only 1 or 2 individuals. Ward \& Hutchings' (1996) analysis of the species dataset is the benchmark for our comparisons.

Multivariate analyses. We used the multivariate analysis package PATN ${ }^{\mathrm{TM}}$ (Belbin 1993) and, following Ward \& Hutchings (1996), calculated Bray \& Curtis dissimilarity measures (Bray \& Curtis 1957) from untransformed abundances for all datasets. It is important to note that the type of data standardisation, data transformation and association measure affect subsequent ordinations (Jackson 1993), and our use of the Bray \& Curtis association measure on untransformed abundance data increases the influence of taxa with high abundances.

Association measures were used to ordinate samples in Semi-strong Hybrid Multidimensional Scaling (SSH). This method uses a nonmetric, ordinal approach above a user-defined association value (Belbin 1991, 1993): that is, dissimilarities above this value are ranked so that the ordination uses only relative dissimilarities. Below this value, the ordination uses a ratio approach. This combination of ordinal and ratio scaling is the principal difference between SSH and metric or nonmetric MDS.

The utility of reduced taxonomic resolution has previously been tested on data from a homogeneous habitat. Our samples came from different habitats as well as from along a pollution gradient. We expected to detect 
patterns associated with both factors. We tested the validity of our interpretation of the results of SSH analyses by conducting randomisation tests (using the ASIM module of the PATN software) that are based on the concept underlying analyses of variance.

Data manipulations. To decrease taxonomic resolution, we pooled data to genus, family, order and class level. The resulting datasets contained 183 genera, 108 families, 46 orders and 15 classes.

We manipulated the species, genus and family datasets to simulate the changes in the dataset characteristics that occurred as we pooled data. Firstly, within a taxonomic level, we created datasets with different attribute:object ratios but with the same non-zeros ratio. We did this by randomly extracting selected numbers of taxa from the species, genus and family datasets. The order and class datasets contained too few taxa for these procedures. The number of taxa extracted was varied to give different attribute:object ratios, and we used 10 different subsets for each number of taxa. Secondly, we increased the non-zeros ratio without changing the attribute:object ratio. We did this by progressively removing the taxa that occurred in the least number of samples in the species and genus datasets (i.e. first the taxa that occurred in only 1 sample, then taxa that occurred in only 2 samples, and so on). From each of these datasets, we generated 10 randomly selected subsets of equal size.

Statistical comparisons. Clarke (1993) suggested that correlations of association matrices provide a natural measure of correspondence between ordinations. We assessed similarity between association matrices by using Mantel tests (Manly 1991, Belbin 1993) based on Pearson's product moment correlation coefficients (r). The Mantel test used in PATN'M compares correlations of original association matrices with correlations arising from 5000 random permutations of the values in each association matrix. As the conclusions arising from tests on original and ranked matrices were the same, we have reported only tests on ranked matrices.

We used SSH stress (Belbin 1993) as a measure of how changing taxonomic resolution and manipulating dataset characteristics affected ordinations. Higher stress values indicate that an ordination plot is less representative of the original distances between samples. Firstly, we used SSH stress for ordinations of each taxonomic resolution in 2, 3, 4 and 5 dimensions to determine the optimal number of dimensions for our analyses. We also examined changes in SSH stress among ordinations based on different taxonomic resolutions, different attribute:object ratios and different non-zeros ratios.
We used Procrustes rotation (Gower 1971) to compare ordinations. Our goodness-of-fit measure was the error sum of squares value generated by PATNTM (Belbin 1993, herein termed $\mathrm{m}^{2}$ ). This measure corresponds to Gower's (1971) sum of squared distances and the $m^{2}$ statistic of Digby \& Kempton (1987) and Jackson (1993). We standardised Procrustes rotations to scale the $\mathrm{m}^{2}$-values from 0 (perfect match) to 1 (no match). Procrustes rotations helped us choose among 2, 3,4 and 5 dimensions for ordinations and to compare ordinations generated from datasets with different taxonomic resolutions, different attribute:object ratios and different non-zeros ratios to the species-level ordination. The species-level ordination was chosen as the target for Procrustes rotations because we focused on the effects of reducing taxonomic resolution.

Monte Carlo randomisation tests allowed us to investigate the significance of SSH stress and $m^{2}$ statistics. Data matrices from each taxonomic level were subjected to Monte Carlo re-ordering in PATN'M (Belbin 1993). The resulting 1000 matrices were analysed using SSH ordination and Procrustes rotation.

\section{RESULTS}

\section{Choice of dimension}

The number of dimensions is significant in MDS ordination techniques because an ordination is recalculated when the number of dimensions is changed, unlike in methods such as Principal Components Analysis, where additional dimensions do not change the orientation of those already calculated. Selecting too few dimensions in MDS may lead to a poor representation of data, and selecting too many dimensions can result in unnecessary noise. In our analyses, both SSH stress and the $\mathrm{m}^{2}$ statistic indicated that the optimum was 3 dimensions (Table 1); therefore, all subsequent analyses were conducted in 3 dimensions.

Table 1 . Changes in SSH stress and error sums of squares $\left(\mathrm{m}^{2}\right)$ with changes in number of dimensions of ordination. -: not applicable, as the species-level ordination was not rotated to itself

\begin{tabular}{|c|c|c|c|c|c|c|}
\hline \multirow{3}{*}{$\begin{array}{l}\text { Taxonomic } \\
\text { level }\end{array}$} & \multicolumn{6}{|c|}{ Change in measure with change in dimension } \\
\hline & \multicolumn{3}{|c|}{ SSH stress } & \multicolumn{3}{|c|}{ Error sums of squares $\left(\mathrm{m}^{2}\right)$} \\
\hline & $2-3$ & $3-4$ & $4-5$ & $2-3$ & $3-4$ & $4-5$ \\
\hline Species & 0.0652 & 0.0241 & 0.0117 & - & - & - \\
\hline Genus & 0.0642 & 0.0249 & 0.0161 & -0.014 & -0.042 & -0.002 \\
\hline Family & 0.0696 & 0.0351 & 0.0249 & 0.375 & $\hat{0} . \hat{0} 4 \hat{0}$ & $-\hat{0} .10 \hat{0}$ \\
\hline Order & 0.0573 & 0.0336 & 0.0239 & 0.227 & -0.048 & 0.012 \\
\hline Class & 0.0435 & 0.0327 & 0.0183 & 0.103 & 0.006 & 0.046 \\
\hline
\end{tabular}




\section{Reducing taxonomic resolution}

Association matrices generated from data at genus, family and order resolutions were highly correlated to the species-level association matrix, but the strength of the correlation was declining (Fig. 1). At class level, the correlation was relatively poor. As Mantel tests did not result in $r$-values greater than those generated with the original association matrices, these results suggest that all association matrices contained non-random information, but the similarity of this information to that in the species-level data decreased.

Although weakening correlations indicated that dissimilarities between samples changed with decreased taxonomic resolution, the influence of such changes was less apparent in the ordination plots (Fig. 2). As the initial orientation of each ordination was arbitrary, we rotated the plots in 3 dimensions using MacSpin ${ }^{T M}$ (Donohu ei di. 1985j to highlight similanties in patterns across taxonomic levels. One dominant pattern was for samples to group according to habitat types (Fig. 2). The groups were relatively compact and separate at the species, genus, family and order levels, but at class level, the groups were more diffuse and overlapping. Within habitat types, the unvegetated intertidal sites and the Zostera intertidal sites on the metal-contaminated transects (outlined I and $Z$ in Fig. 2) were separated from sites on unpolluted transects (solid I and $Z$ in Fig. 2).

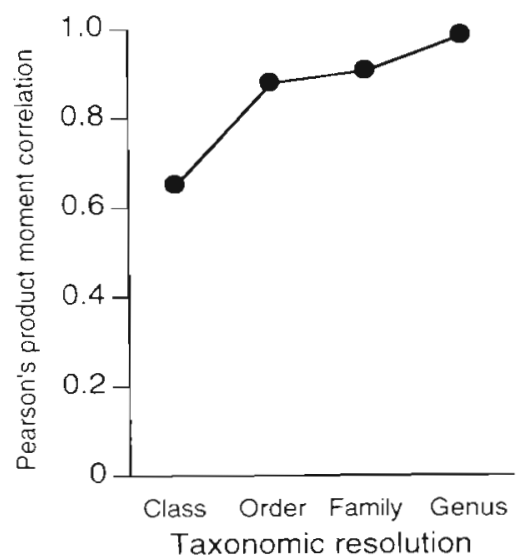

Fig. 1. Pearson's product moment correlation values (r) for correlations between ranked association matrices at different levels of taxonomic resolution to the ranked species level association matrix

ASIM tests on the results of all SSH analyses supported our interpretation that sites in different habitats and sites with different levels of contamination were separated. For all taxonomic levels, none of the 1000 randomised results provided a better interpretation of the SSH results than the one we chose.

The SSH stress values for these ordinations revealed a complex pattern (Fig. 3). SSH stress was highest at the family and order levels and lowest at the class level
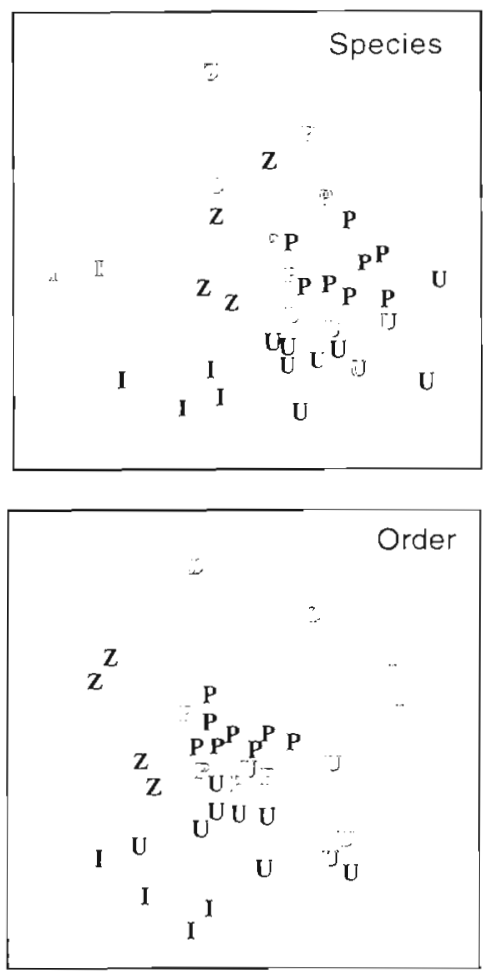
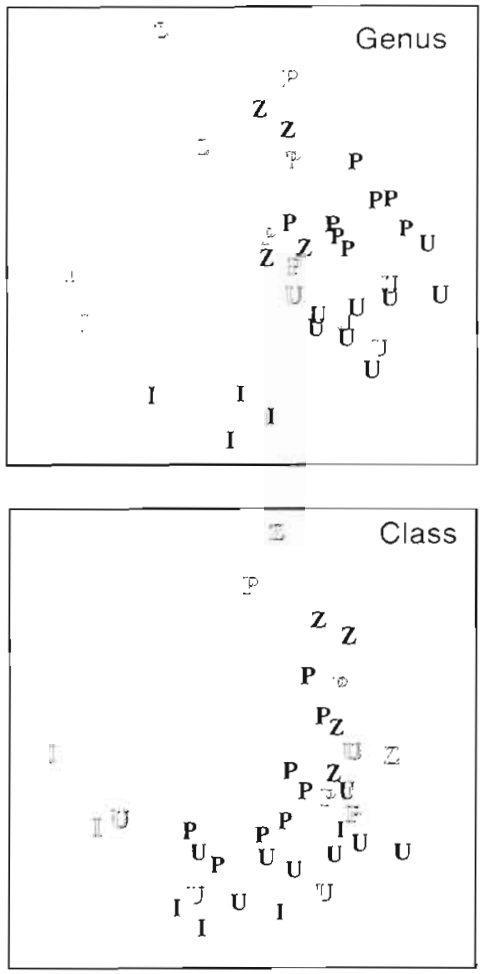

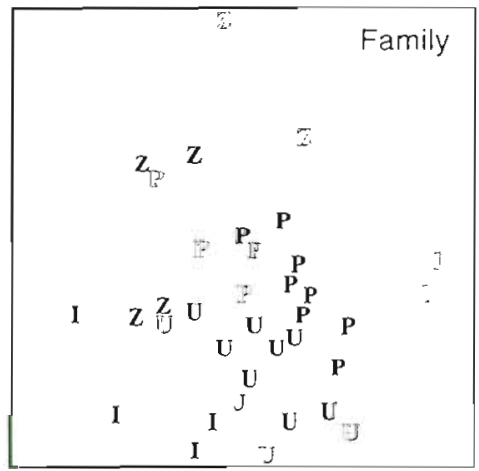

Fig. 2. SSH plots generated from data at species, genus, family, order and class levels. Sample codes: (I) Intertidal Unvegetated; (Z) Zosterd; (P) Posidonia; (U) Subtidal Unvegetated; samples in outline are from the polluted sites. Ordinations have been rotated with the MacSpin ${ }^{\text {TM }}$ software so that patterns can be clearly vlewed in 2 dimensions 
(Fig. 3). In contrast, mean SSH stress for the ordinations created by Monte Carlo randomisations did not increase at the family and order levels (Fig. 3). Stress in the random ordinations also decreased most at the class level (Fig. 3). The SSH stress values for the real data were lower than the mean values generated from random datasets, but the original stress values approached and, at class level, entered the tail of the frequency distribution for the random datasets (Figs. 3 \& 4). It appeared that the real data always contained information that was non-random, but the amount of information decreased with decreasing taxonomic resolution.

Procrustes rotations showed that the $\mathrm{m}^{2}$ statistic increased steadily with decreasing taxonomic resolution, which meant that ordinations at lower taxonomic resolutions were less similar to the original specieslevel plot (Fig 5). Procrustes rotations for 1000 randomised datasets at each taxonomic resolution yielded mean $\mathrm{m}^{2}$ statistics that were consistently higher than those from the original datasets (Fig. 5). The $m^{2}$ statistics for the original data approached but did not enter the frequency distribution of random $\mathrm{m}^{2}$ as taxonomic resolution decreased (Fig. 6). Once again, the original data contained non-random information at all taxonomic levels, but the amount of non-random information decreased with lower taxonomic resolution. It is important to note that the mean $\mathrm{m}^{2}$ statistics from the randomisations exhibited no relationship to changes in the size of datasets (Fig. 5).

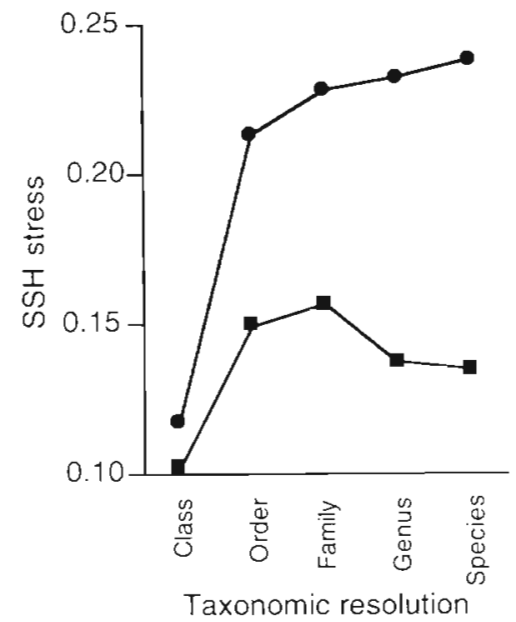

Fig. 3. SSH stress for ordinations at increasing levels of taxonomic resolution. ( $\square)$ original data; ( $)$ mean from Monte Carlo randomisations $(\mathrm{n}=1000)$

\section{Manipulating dataset characteristics}

Among subsets of species and genus datasets with constant non-zeros ratios, the SSH stress and the $\mathrm{m}^{2}$ statistic increased as the attribute:object ratio decreased (Fig. 7). The results from subsets of the family-level dataset showed a less clear pattern (Fig. 7). The nonzeros ratios did not differ greatly among the subsets
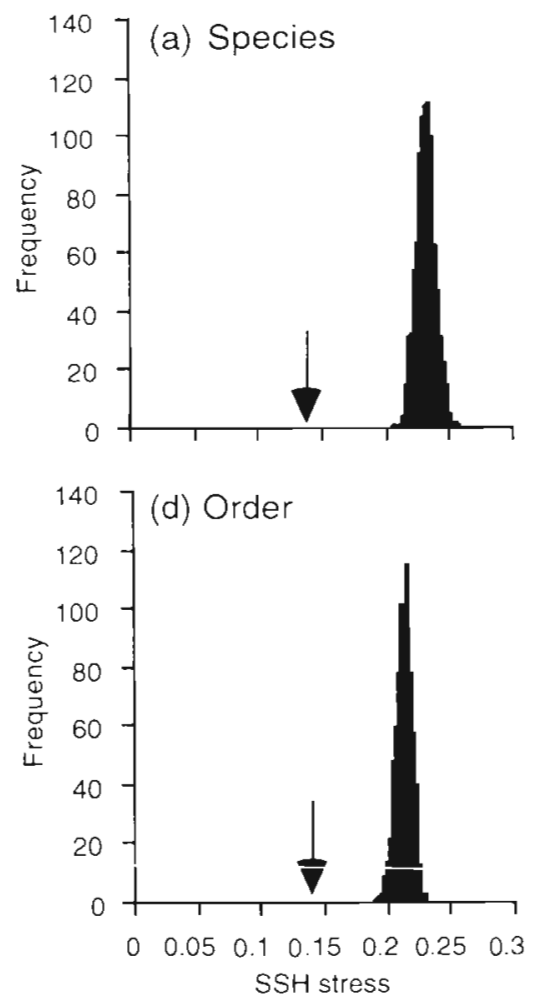

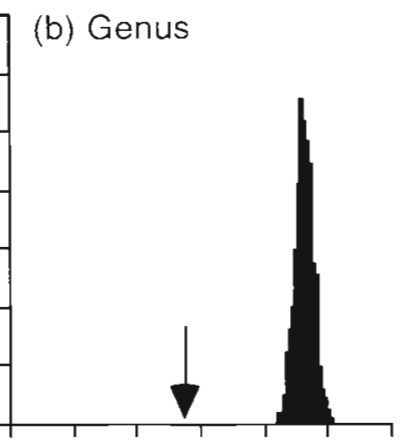

(e) Class

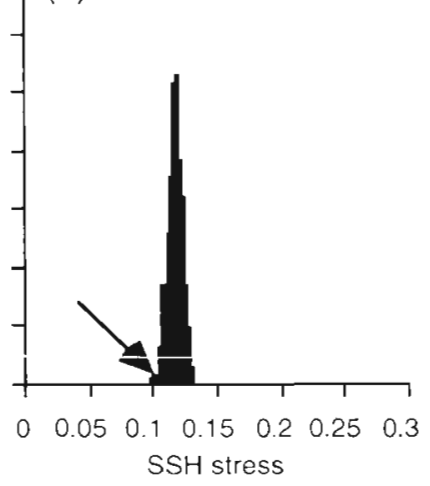

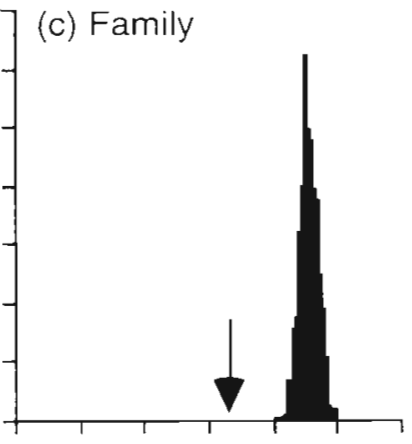

$\begin{array}{lllllll}0 & 0.05 & 0.1 & 0.15 & 0.2 & 0.25 & 0.3\end{array}$

Fig. 4. Frequency distributions of SSH stress

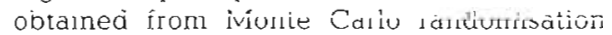
procedures for each taxonomic resolution. Arrows indicate the SSH stress values from the original data 


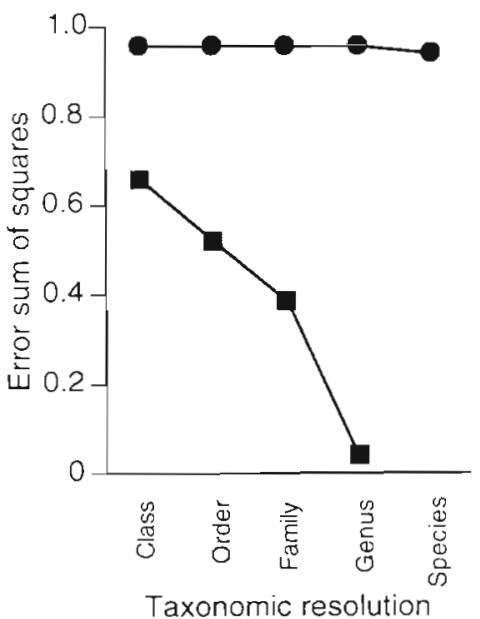

Fig. 5. Error sum of squares statistics $\left(\mathrm{m}^{2}\right)$ for Procrustes rotations of ordinations at increasing taxonomic resolutions to the species level. (W) original data; (1) mean from Monte Carlo randomisations $(\mathrm{n}=1.000)$

within each taxonomic level (Fig. 7). These trends indicate less fit between association matrices and ordinations as the attribute:object ratio was reduced and less correspondence between ordinations based on datasets with fewer attributes than the species-level target ordination. The non-zeros ratios for genus datasets was higher than the non-zeros ratios for species datasets (legend to Fig. 7), and the SSH stress and $\mathrm{m}^{2}$ for genus data were lower than those for species data (Fig. 7).

When the attribute:object ratio remained constant, both SSH stress and $m^{2}$ statistics decreased as the nonzeros ratio increased (Fig. 8). These trends were apparent for subsets of both species and genus datasets, and they indicate a better fit of ordinations to association matrices and a better correspondence between ordinations and the species-level target ordination.

\section{DISCUSSION}

The samples comprising our dataset were taken from different habitats along a pollution gradient surrounding a lead smelter. Their patterns of species composition are related to habitat and metal pollution (Ward \& Young 1981, Ward \& Hutchings 1996; our results). The information contained in these samples provides a useful starting point for testing hypotheses about the effectiveness of analyses at reduced taxonomic resolutions.

Monte Carlo randomisations at each taxonomic resolution tested the effect of changing dataset size by blurring any pattern inherent in the data. Our results showed that SSH stress could be expected to decrease with decreasing dataset size and $\mathrm{m}^{2}$ statistics could be expected to remain unchanged. From these results, we
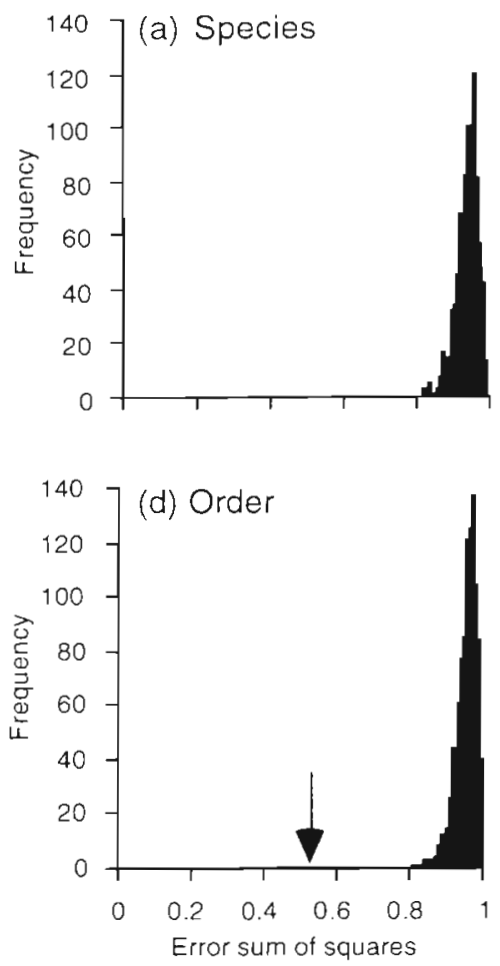
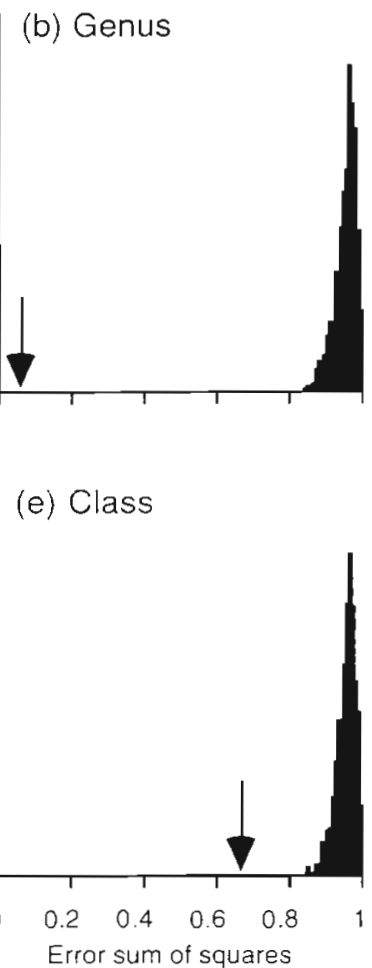

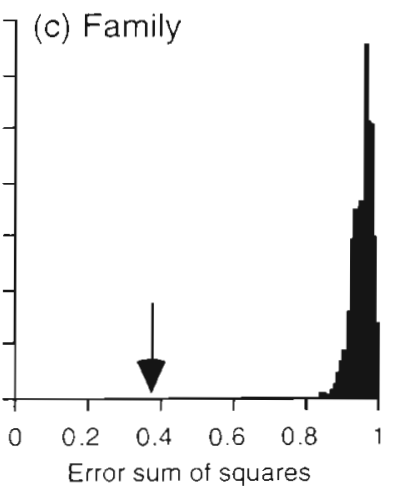

Fig. 6. Frequency distributions of the error sum of squares statistic $\left(\mathrm{m}^{2}\right)$ generated by Procrustes rotations of Monte Carlo randomisations to the original species level ordination. Arrows indicate the $\mathrm{m}^{2}$ statistics from Procrustes rotations of the original data. There is no arrow in the frequency distribution at spe. cies level because this was the target 

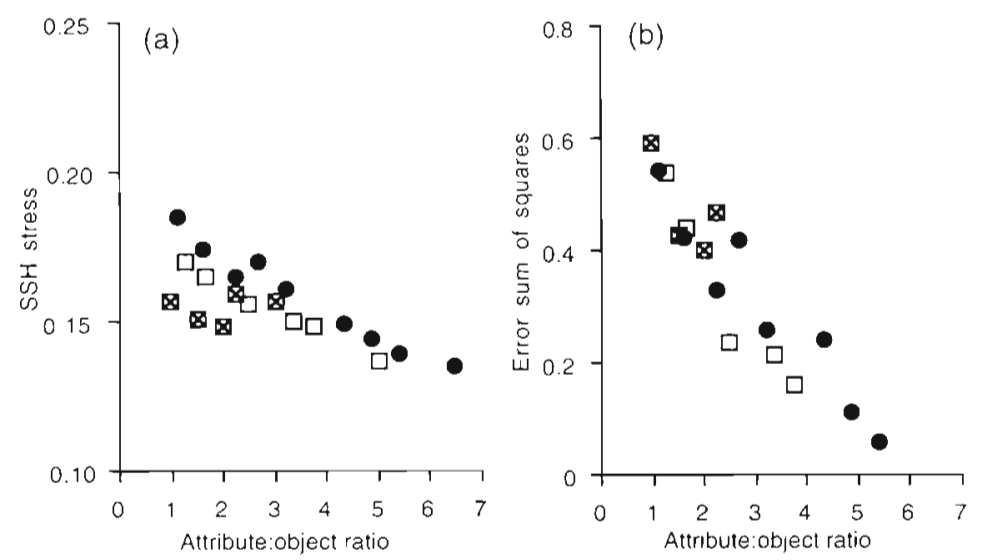

Fig. 7. Mean (a) SSH stress and (b) error sum of squares statistics $\left(m^{2}\right)$ for randomly generated subsets of data $(n=10)$ versus attribute:object ratio. The target for Procrustes rotations was the species-level ordination. (-) Species (range of non-zeros ratio $=6.58-7.01$ ); $(\square)$ genus (range of nonzeros ratio $=7.36-7.94) ;(\bigotimes)$ family (range of non-zeros ratio $=9.45-9.88$ )

These opposing effects may have led to the changes in SSH stress and $\mathrm{m}^{2}$ statistics that we observed as we manipulated the real data. The observed increase in SSH stress at the family and order levels followed the trend accompanying decreasing attribute:object ratios, and the decrease in SSH stress at the class level followed the trend accompanying increasing non-zeros ratios. For these data, the non-zeros ratio may be the dominant influence on SSH stress above order level and the attribute:object ratio may control SSH stress below order level. In contrast, $\mathrm{m}^{2}$ statistics only followed the trend accompanying decreasing attribute:object ratios.

Despite the changes in SSH stress and $\mathrm{m}^{2}$ statistics, 2 patterns remained visible in ordinations to the order level. These 2 patterns (separation of sites according to habi-

would predict that pooling data to lower taxonomic resolutions (reducing the dataset size) would lead to a decrease in SSH stress and no change in $m^{2}$ statistics. These trends suggest a better fit between multivariate measures of distance and ordinations and no change in patterns in ordinations.

Manipulations of real data did not yield these patterns. SSH stress increased slightly at the family and order levels and $\mathrm{m}^{2}$ statistics increased steadily at lower taxonomic resolutions. These results indicate less fit between ordinations and underlying measures of distance and changes in the patterns in ordinations. In addition, changes in taxonomic resolution led to less similar association matrices as shown by decreasing correlations between matrices. Log-transformed data revealed the same patterns, suggesting that this difference was not caused by characteristics associated with the application of Bray \& Curtis association measures to untransformed abundance data (authors' unpubl. data).

In addition to lowering overall dataset size, decreasing taxonomic resolution also decreases the attribute:object ratio and increases the non-zeros ratio in the resulting datasets. When the attribute:object ratio was decreased independently of the nonzeros ratio, both SSH stress and the $m^{2}$ statistic generally increased. Conversely, when the non-zeros ratio was increased and the attribute:object ratio was held constant, buth the SSH stross and the $m^{2}$ statistic decreased. These results indicate that decreasing taxonomic resolution may create opposing effects on ordinations. tat type and separation of the metal-contaminated intertidal sites of Transect A from the intertidal sites of Transect B and C) are those discussed by Ward \& Hutchings (1996). In addition, SSH stress and $\mathrm{m}^{2}$ statistics always remained lower than the mean values from Monte Carlo randomisations, confirming the presence of non-random information in the real data from all taxonomic levels.

Visual comparisons of our MDS ordination plots generally support arguments that analyses of data at lower taxonomic resolutions generate distribution patterns associated with pollution gradients as clearly as analyses at species level (Heip et al. 1988, Warwick 1988a, b, Gray et al. 1990, Agard et al. 1993, Warwick \& Clarke 1993). Our results also indicate that analyses based on
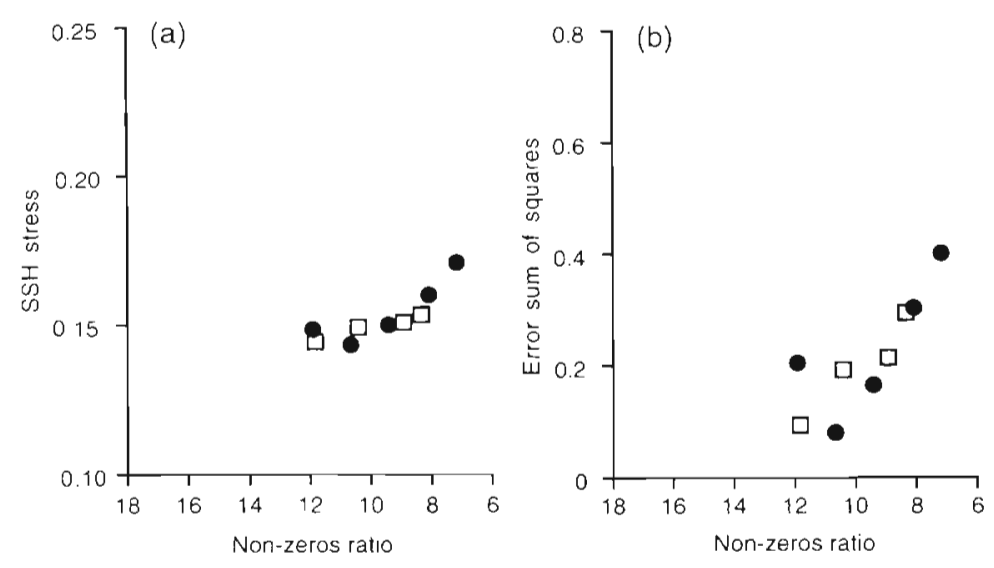

Fig. \&. Mcan (a) SSH stross and (b) error sum of squares statistics $\left(m^{2}\right.$ ) for randomly generated subsets of data $(n=10)$ versus the non-zeros ratio. The target for Procrustes rotations was the species level ordination. (-) species; ( $\square$ ) genus 
reduced taxonomic resolutions may detect patterns associated with habitat gradients. The ability to detect a 'signal' associated with pollution gradients amid the 'noise' associated with a habitat gradient increases the utility of analyses based on reduced taxonomic resolutions.

It is important to note there are changes associated with decreasing the level of taxonomic resolution. Lower correlations between association matrices indicate a change in the estimates of distance between samples. Higher SSH stress values at some taxonomic levels indicate that ordinations are less successful at representing the distances between samples. Higher $\mathrm{m}^{2}$ statistics indicate that ordinations based on data at lower taxonomic resolutions are less similar to ordinations of species-level data.

Overall, visual comparisons of MDS plots are rather subjective tests. Visual comparisons have no objective way of estumating resolving power, i.e. no mcans of estimating the probabilities of proposing the existence of a pollution or habitat gradient that does not exist or failing to detect a gradient that does exist. Our results indicate that the arbitrary selection of taxonomic resolution may alter the risk of making an error with no guarantee that the risk will be increased or decreased. Therefore, results from a single study that suggest certain levels of taxonomic resolution are adequate or preferable for detecting the impacts of pollution may not be broadly applicable.

Before reduced taxonomic resolution is widely used, there is a need to explore the generality of our findings with other datasets. Different assemblages collected along different gradients may yield other results. For example, assemblages along weaker natural gradients may not yield robust patterns like those seen along a strong pollution or habitat gradient. The influences reducing taxonomic resolution and altering the attribute:object or non-zeros ratios warrant evaluation because of the need to balance the cost of sampling a large number of places for data at the species level against the loss of potentially important information associated with taking fewer samples (reducing the number of objects) and analysing data at lower taxonomic resolutions.

Acknowledgements. This work was funded by the Marine Environment Research Program in the CSIRO Division of Fisheries. The original field sampling was funded by the International Lead Zinc Research Organization (New York), and the fauna was identified by Pat Hutchings and the staff of the Australian Museum (Sydney). We are very grateful to Dennis Heinemann (CSIRO Division of Fisheries) for his assistance in preparing the scripts that generated the series of Monte Carlo randomisations. We are also grateful to Lee Belbin (CSIRO Division of Wildlife and Ecology), David Fox (CSIRO Biometrics Unit) and Dennis Heinemann for their critical reviews of the manuscript.

\section{LITERATURE CITED}

Agard JBR, Gobin J, Warwick RM (1993) Analysis of marine macrobenthic community structure in relation to pollution. natural oil seepage and seasonal disturbance in a tropical environment (Trinidad, West Indies). Mar Ecol Prog Ser 92:233-243

Austen MC, Warwick RM, Rosado MC (1989) Melobenthic and macrobenthic community structure along a putative pollution gradient in southern Portugal. Mar Pollut Bull 20:398-405

Belbin L (1991) Semi-strong hybrid scaling, a new ordination algonthm. J Veg Sci 2:491-496

Belbin L (1993) PATN technical reference. CSIRO Division of Wildlife and Ecology, Canbera

Bray JR, Curtis JT (1957) An ordination of the upland forest communities of southern Wisconsin. Ecol Monogr 27: $325-349$

Cl.arke KR (1993) Non-parametric multivariate analyses of changes in community structure. Aust J Ecol 18:117-143

Digby PGN, Kempton RA (1987) Multivariate analysis of ecological communities. Chapman \& Hall, London

Donoho AW, Donoho DL, Gasko M, Oison CW (1985) MacSpin graphical data analysis software. D2 Software, Austin

Ellis D (1985) Taxonomic sufficiency in pollution assessment. Mar Pollut Bull 16:459

Ferraro SP, Cole FA (1990) Taxonomic level and sample size sufficient for assessing pollution impacts on the Southern Californa Bight macrobenthos. Mar Ecol Prog Ser 67: $251-262$

Gower JC (1971) Statistical methods of comparing different multivariate analyses of the same data. In: Hodson FR, Kendall DG, Tautu P (eds) Mathematics in the archaeological and historical sciences. Edinburgh University Press, Edinburgh, p 138-149

Gray JS, Aschan M, Carr MR, Clarke KR, Green RH, Pearson TH. Rosenberg R, Warwick RM (1988) Analysis of community attributes of the benthic macrofauna of Frierfjord/ Langesundfjord and in a mesocosm experiment. Mar Ecol Prog Ser 46:151-165

Gray JS, Clarke KR, Warwick RM, Pearson TH, Hobbs G (1990) Detection of initial effects of pollution on marine benthos: an example from the Ekofisk and Eldfisk oilfields, North Sea. Mar Ecol Prog Ser 66:285-299

Heip C, Warwick RM, Carr MR, Herman PMJ, Huys R, Smol N, Van Holsbeke K (1988) Analysis of community attributes of the benthic meiofauna of Frierfjord/Langesundfjord. Mar Ecol Prog Ser 46:171-180

Jackson DA (1993) Multivariate analysis of benthic invertebrate communities: the implication of choosing particular data standardisations, measures of association, and ordination methods. Hydrobiologia 268:9-26

Manly BFJ (1991) Randomisation and Monte Carlo methods in biology. Chapman \& Hall, London

Rees HL (compiler) (1993) Analysis and interpretation of benthic community data at sewage sludge disposal sites. Aquat Env Monit Rep, vo. 37. MAFF Direct Fish Res, Lowestoft

Roper DS, Smith DG, Read GB (1989) Benthos associated with two New Zealand coastal outfalls. NZ J Mar Freshwat Res 23:295-309

Stull JK, Haydock CI, Smith RW, Montagne DE (1986) Longterm changes in the benthic community on the coastal shelf of Palos Verdes, Southern California. Mar Bıol 91: $539-551$

Ward TJ, Hutchings PA (1996) Effects of trace metals on infaunal species composition in polluted intertidal and 
subtidal marine sediments near a lead smelter, Spencer Gulf, South Australia. Mar Ecol Prog Ser 135:123-135

Ward TJ, Young PC (1981) Trace metal contamination of shallow marine sediments near a lead smelter, Spencer Gulf, South Australia. Aust J Mar Freshwat Res 32:45-56

Ward TJ, Young PC (1982) Effects of sediment trace metals and particle size on the community structure of epibenthic seagrass fauna near a lead smelter, South Australia. Mar Ecol Prog Ser 9:137-146

Warwick RM (1988a) Analysis of community attributes of the macrobenthos of Frierfjord/Langesundfjord at taxonomic levels higher than species. Mar Ecol Prog Ser 46:167-170

Warwick RM (1988b) The level of taxonomic discrimination required to detect pollution effects on marine benthic communities. Mar Pollut Bull 19:259-268

This article was presented by J. Gray (Senior Editorial Advisor), Oslo, Norway
Warwick RM (1993) Environmental studies on marine communities: pragmatical considerations. Aust J Ecol 18: $63-80$

Warwick RM. Clarke KR (1993) Comparing the severity of disturbance: a meta-analysis of marine macrobenthic community data. Mar Ecol Prog Ser 92:221-231

Warwick RM, Platt HM, Clarke KR, Agard J, Gobin J (1990) Analysis of macrobenthic and meiobenthic community structure in relation to pollution and disturbance in Hamilton Harbour, Bermuda. J Exp Mar Biol Ecol 138: $119-142$

Zenetos A, Papathanassiou E (1989) Community parameters and multivariate analysis as a means of assessing the effects of tannery effluents on macrobenthos. Mar Pollut Bull 20:176-181

Manuscript first received: March 20, 1995

Revised version accepted: October 24, 1995 